Ada Ávila Assunção

(D) https://orcid.org/0000-0003-2123-0422

Mariana Roberta Lopes Simões ${ }^{b}$

(D) https://orcid.org/0000-0003-0543-6906

Emanuella Gomes Maiac

(D) https://orcid.org/0000-0001-6655-0230

Marcus Alessandro Alcantara ${ }^{\mathrm{d}}$

(DD http://orcid.org/0000-0001-9233-0186

Renata Jardime

(D) https://orcid.org/0000-0003-2760-3664

a Universidade Federal de Minas Gerais (UFMG), Faculdade de Medicina, Departamento de Medicina Preventiva e Social. Belo Horizonte, MG, Brasil.

bUniversidade Federal dos Vales do Jequitinhonha e Mucuri (UFVJM), Departamento de Enfermagem, Curso de Enfermagem. Diamantina, MG, Brasil.

'Universidade Estadual de Santa Cruz (UESC), Departamento de Ciências da Saúde, Curso de Enfermagem. Ilhéus, BA, Brasil.

'Universidade Federal dos Vales do Jequitinhonha e Mucuri (UFVJM), Departamento de Fisioterapia, Curso de Fisioterapia. Diamantina, MG, Brasil.

eUniversidade Federal de Sergipe (UFS), Departamento de Educação em Saúde. Lagarto, SE, Brasil.

Contato:

Ada Ávila Assunção

E-mail:

adavila@medicina.ufmg.br

Os autores declaram que o trabalho não foi subvencionado e que não há conflitos de interesses.

Os autores informam que o trabalho não foi apresentado em evento científico.

\section{COVID-19: estudo de protocolos de proteção individual para profissionais da saúde}

\author{
COVID-19: a study of personal protection \\ protocols for health workers
}

\section{Resumo}

Objetivo: realizar comparação interpaíses e entre estados brasileiros quanto ao conteúdo dos protocolos de proteção para profissionais de saúde que atuam na assistência aos doentes da COVID-19 e desenvolver análise crítica ao modelo de prevenção que adota indicação e uso de equipamentos de proteção individual (EPI) como resposta única a um problema de caráter multidimensional. Métodos: estudo exploratório com base em revisões disponíveis na biblioteca Cochrane, articuladas com a análise dos protocolos nacionais de Argentina, Brasil, China e Estados Unidos da América e as normas previstas nos estados do Amazonas, Bahia, Minas Gerais e São Paulo, todos selecionados por critérios de conveniência. Resultados: observou-se dissensos quanto aos tipos de proteção recomendados. Somente na China eram indicados respiradores de alta eficiência de filtragem, além de modelos para o rosto inteiro nos casos de procedimentos invasivos. O reuso de equipamentos não é indicado, mas estava autorizado no protocolo brasileiro. Quanto aos dispositivos de vestuário, também não há convergência. Conclusão: os resultados reforçam a necessidade de revisão dos protocolos de proteção dos profissionais da saúde que atuam no enfrentamento da Covid-19. Ações em busca de debate institucional, interpaíses e interestaduais sobre modelos de prevenção são essenciais para alcançar consistência nas recomendações.

Palavras-chave: covid-19; pessoal de saúde; equipamento de proteção individual; saúde do trabalhador.

\begin{abstract}
Objective: to make a comparison between countries and between Brazilian states regarding the contents of protection protocols for health workers assisting COVID-19 patients and to undertake a critical analysis of the prevention model that adopts the recommendation and use of personal protective equipment (PPE) as the only response to a multidimensional problem. Methods: this is an exploratory study based on revisions available at the Cochrane Library, articulated with the analysis of the national protocols of Argentina, Brazil, China and the United States of America and those of the states of Amazonas, Bahia, Minas Gerais and São Paulo, all selected by convenience criteria. Results: there were differences bewteen the recommended types of protection. Only China recommended high filtration efficiency respirators as well as pieces covering the whole face for invasive procedures. Reusing the equipment is not recommended, but it was authorized in the Brazilian protocol. There was also no concurrence about clothing devices. Conclusion: the results reinforce the need for revision of the protocols for the protection of health workers working in the COVID-19 effort. Actions to promote institutional, inter-country and interstate debate on prevention models are essential for achieving consistency in the recommendations.
\end{abstract}

Keywords: COVID-19; health personnel; personal protective equipment; occupational health. 


\section{Introdução}

A ameaça de contágio pela COVID-19 (Síndrome Respiratória Aguda Grave pelo SARS-CoV-2) alterou a rotina das cidades e suas economias, além de ter potencializado a pressão, por vezes intransponível, sobre os sistemas de saúde. O volume das demandas se mostrou incompatível com a capacidade do sistema em algumas localidades, gerando desassistência e perturbação no fluxo rotineiro do atendimento. Desde os primeiros surtos, os profissionais de saúde foram atingidos, fosse pela infecção ou pelo estresse mental e social de que são vítimas ${ }^{1}$. Vale mencionar o risco máximo dos que estão na linha de frente, porque lidam com pacientes assintomáticos e sintomáticos, testados ou não².

A maior parte das tarefas realizadas pelo pessoal que assiste aos pacientes envolve contato direto, de maneira a justificar protocolos específicos de proteção ${ }^{3}$. Há carência de recursos materiais e de expertise para reverter a situação de vulnerabilidade na linha de frente da assistência a esses pacientes ${ }^{2}$.

Em Wuhan, na China, a probabilidade de infecção entre os profissionais dos serviços de saúde foi três vezes maior que na população geral. Em outros países atingidos pela pandemia, foram registrados afastamentos para tratamento ou quarentena, falecimentos, sofrimento das comunidades e desequilíbrios na organização ${ }^{1}$.

Como resposta, deu-se ênfase ao fornecimento de equipamentos de proteção individual (EPI), que são vestimentas e aparatos usados para proteção contra agentes que possam oferecer riscos à saúde e à segurança no trabalho. Respiradores, máscaras, luvas, óculos de proteção, protetores faciais, capotes, aventais, revestimentos para cabeça e calçados são os mais utilizados. Esses equipamentos são cruciais, ainda que insuficientes, para proteger os profissionais da saúde ${ }^{4}$.

A situação pandêmica exige desses profissionais a adoção de tais medidas com objetivo de autoproteção, concomitante à proteção dos outros em um contexto de alto risco ${ }^{5}$. O EPI tem a função de barreira contra o agente patógeno na região da face, dos olhos, das mãos e outras partes do corpo ${ }^{6}$. O uso faz parte das recomendações do Ministério da Saúde (MS) no Brasil ${ }^{7}$ e da Organização Mundial da Saúde $(\mathrm{OMS})^{8}$, visando a redução da transmissão do vírus no ambiente dos serviços de saúde.

A indicação de uso do EPI nos serviços de assistência à saúde é uma medida draconiana, ou seja, soberana e absoluta, porque o risco é de se contrair a própria doença que o profissional busca tratar e curar.

A baixa adesão ao uso desses equipamentos não é um dilema recente. Em 1990, o artigo "Precauções universais são universalmente ignoradas"9 apresentou importantes esclarecimentos sobre essa realidade. Durante a epidemia da gripe influenza H1N1, o problema foi identificado em quatorze hospitais canadenses ${ }^{10} \mathrm{e}$ em unidades de tratamento intensivo de dezessete províncias da China ${ }^{11}$. Se está relativamente documentada a relevância da intensificação de medidas de autoproteção em situações de doenças infectocontagiosas fatais ${ }^{12}$, como explicar os registros de baixa adesão ao uso dos EPI?

Desde os primeiros surtos da COVID-19, protocolos foram publicados e atualizados, tanto para proteger a saúde dos profissionais da saúde quanto para mitigar a disseminação do novo coronavírus. Qual é a abrangência das medidas recomendadas nos protocolos vigentes no Brasil para proteger os profissionais de saúde? Há consenso entre os países na prescrição de EPI conforme seus protocolos?

Para responder às três perguntas formuladas acima, o objetivo do presente estudo foi realizar uma comparação interpaíses e entre estados brasileiros quanto ao conteúdo dos protocolos de proteção para profissionais de saúde que atuam na assistência aos doentes da COVID-19. Será desenvolvida análise crítica ao modelo de prevenção que adota a indicação e uso de EPI como resposta única a um problema de caráter multidimensional.

\section{Métodos}

\section{Delineamento e procedimentos técnicos}

O material empírico que se apresenta é fruto da pesquisa exploratória que contemplou uma análise de dois conjuntos de informações. O primeiro diz respeito aos protocolos institucionais publicados nos portais da OMS, dos MS de quatro países (Argentina, Brasil, China e Estados Unidos da América - EUA) e das secretarias estaduais de quatro estados federativos do Brasil (Amazonas, Bahia, Minas Gerais e São Paulo). O segundo conjunto foi extraído de revisões bibliográficas sistematizadas no dossiê sobre a prevenção da COVID-19 produzido e atualizado pela biblioteca Cochrane ${ }^{13}$ entre março e maio de 2020.

Em termos operacionais, a pesquisa foi delineada em quatro etapas. A primeira consistiu no estudo do protocolo da OMS para identificar os eixos das medidas de proteção que constituíram a planilha de critérios para, na segunda etapa, estudar as recomendações vigentes nos países selecionados. A terceira etapa foi a comparação entre os protocolos estaduais brasileiros. O quarto percurso correspondeu a um exercício de identificação das evidências científicas quanto aos fatores relacionados à adesão ao uso de EPI, bem como os limites conhecidos de sua eficácia 
para a prevenção da infecção em profissionais dos serviços de saúde. Foram tomadas como base as revisões da biblioteca Cochrane ${ }^{13}$ com intuito de identificar resultados relacionados às barreiras, facilitadores e eficácia do uso de EPI para proteger os profissionais da saúde expostos ao novo coronavírus.

A coleta de dados foi realizada por dois autores de forma independente. Os rascunhos produzidos foram avaliados pelos demais autores do artigo.

\section{Critérios para identificação e seleção do material empírico}

O protocolo da China $^{14}$ foi de pronto incluído em razão de seu esforço pioneiro na produção de resultados sobre a avaliação da eficácia dos EPI no contexto da crise, o que está refletido na expressiva participação dos autores daquele país na literatura consultada ${ }^{13}$. As recomendações dos institutos especializados em higiene ocupacional dos EUA ${ }^{15}$ têm sido referência para a produção de normas técnicas, além de orientarem a regulamentação infralegal nesse âmbito, no Brasil ${ }^{6}$, e por isso foram incluídas. A Argentina ${ }^{16}$ foi selecionada porque, até maio de 2020, estava entre os países da América do Sul com menor incidência de casos.

Foram selecionados por conveniência protocolos dos estados da Bahia ${ }^{17}$, Minas Gerais ${ }^{18}$, São Paulo $^{19}$ e Amazonas ${ }^{20}$, sendo esta última escolha em razão do colapso do sistema de saúde ocorrido neste estado à época da coleta de dados. As demais unidades federativas correspondem às áreas de atuação dos autores.

Os protocolos foram comparados entre si em suas respectivas instâncias (nacional e estadual) e em relação ao protocolo do MS do Brasil ${ }^{7}$. A tabulação e sistematização dos dados referentes aos tipos de EPI recomendados pelos países e estados brasileiros selecionados foi realizada respeitando a estrutura dos protocolos, de maneira a contemplar o conjunto de itens presente em cada um deles.

Em 4 de março de 2020, foi publicado o primeiro dossiê Coronavírus (COVID-19) da biblioteca Cochrane $^{13}$. Desde então, o material inicialmente disponível em inglês também passou a ser apresentado em chinês simplificado, tcheco, alemão, farsi, japonês, malaio, polonês, português, russo e espanhol. O objetivo desse dossiê especial é ofertar acesso imediato às revisões sistemáticas mais relevantes para o controle da infecção e coerentes com as diretrizes da OMS. A produção foi sistematizada segundo as regiões mais afetadas pela pandemia, além de estar afinada com a produção de três redes específicas: Cochrane Public Health and Health Systems Network; Cochrane Musculoskeletal, Oral, Cutânea e Sensorial Network; e a Cochrane Intensive Care and Emergency Medicine Network. Para este estudo, foram reunidas evidências apresentadas em cinco revisões incluídas no tópico Coronavírus (COVID-19): medidas para o controle e a prevenção de infecções ${ }^{13}$ (Quadro 1).

Quadro 1 Bases de dados das revisões de literatura que compõem o tópico Coronavírus (COVID-19): medidas para o controle e a prevenção de infecções do dossiê especial da biblioteca Cochrane ${ }^{13}$, em 25 de maio de 2020

\begin{tabular}{|c|c|}
\hline Título da revisão da literatura & Bases de dados para a revisão \\
\hline $\begin{array}{l}\text { Intervenções para aumentar a adesão à higiene das mãos durante os cuidados de } \\
\text { saúde ao paciente }{ }^{21}\end{array}$ & $\begin{array}{l}\text { Cochrane Register of Controlled Trials, PubMed, } \\
\text { Embase e CINAHL }\end{array}$ \\
\hline $\begin{array}{l}\text { Como melhorar a aderência dos profissionais de saúde às precauções padrão para } \\
\text { o controle de infecções associadas aos cuidados de saúde }{ }^{22}\end{array}$ & CENTRAL, MEDLINE, Embase, CINAHL, LILACS \\
\hline $\begin{array}{l}\text { Equipamento de proteção individual para profissionais de saúde para } \\
\text { prevenir doenças altamente contagiosas pela exposição a fluidos corporais } \\
\text { contaminados }^{23}\end{array}$ & $\begin{array}{l}\text { CENTRAL, MEDLINE, Embase e CINAHL até } 20 \text { de } \\
\text { março de } 2020\end{array}$ \\
\hline $\begin{array}{l}\text { Barreiras e facilitadores da adesão dos trabalhadores da saúde às diretrizes de } \\
\text { prevenção e controle de infecções (IPC) para doenças infecciosas respiratórias: } \\
\text { uma síntese rápida de evidências qualitativas }{ }^{24}\end{array}$ & COVID MEDLINE em 26 de março de 2020 \\
\hline $\begin{array}{l}\text { Intervenções comportamentais para incentivar o uso de equipamento de } \\
\text { proteção respiratória entre trabalhadores }\end{array}$ & $\begin{array}{l}\text { Cochrane Work Group Specialised Register, Cochrane } \\
\text { Central Register of Controlled Trials (2016), } \\
\text { MEDLINE (1980 a 2016), EMBASE (1980 a 2016) e } \\
\text { CINAHL }\end{array}$ \\
\hline
\end{tabular}

Fonte: Elaboração própria. 
A seleção de evidências citadas nas revisões da biblioteca Cochrane ${ }^{13}$ não foi orientada por eixos pré-estabelecidos. Ao contrário, o curto período - dois meses - passado desde o reconhecimento da situação de crise pandêmica pela OMS, em março de 2020, até a realização do presente estudo justificou assumir o caráter aberto desta investigação exploratória.

\section{Resultados e discussão}

\section{Protocolos para uso de EPI: comparação interpaíses e entre estados do Brasil}

As recomendações sobre quando, qual e como utilizar um EPI têm a ver com o conhecimento sobre as rotas de transmissão do patógeno que se busca debelar ${ }^{11}$. No caso do novo coronavírus, o potencial de transmissão e seus impactos justificam proposições sistêmicas ${ }^{13}$, já que é possível haver contaminação direta ou indireta. A primeira refere-se à transferência direta e imediata dos materiais virais a uma porta de entrada receptiva pela qual se pode consumar a infecção. As situações mais comuns são a projeção direta de gotículas de muco de saliva do sujeito infectado ao espirrar, tossir ou falar para as mucosas nasais e orais do sujeito exposto. Quanto à transmissão indireta, ocorre por meio de um veículo, materiais ou objetos, que servem de meio para conduzir as partículas infectadas para o hospedeiro suscetível.

O conhecimento sobre a viabilidade do vírus em superfícies, contudo, ainda é limitado, enquanto está relativamente esclarecida sua transmissão por meio das gotículas respiratórias $(>5 \mu \mathrm{m})$ e aerossóis $(\leq 5 \mu \mathrm{m})$. A plausibilidade da transmissão por meio de objetos contaminados está apoiada em evidências de viabilidade do novo coronavírus, que provavelmente conserva as propriedades para a infecção durante dias quando depositado nos materiais ${ }^{12,15}$. Até agora, sabe-se que a transmissão inter-humana do novo coronavírus ocorre por meio de aerossóis e gotículas respiratórias e contato direto ou indireto de partes do corpo com esses materiais. As gotículas que carreiam o vírus são expelidas por tosse ou espirros da pessoa já contaminada, atingem o corpo de quem está no ambiente ou se depositam nas superfícies das edificações, instalações, mobiliário e objetos ${ }^{13}$. Aerossóis contendo o vírus são produzidos durante procedimentos realizados na urgência e nas unidades de terapia intensiva, como intubação endotraqueal e aspiração de vias aéreas. Aerossóis são produto de uma combinação de gases respiratórios expirados, partículas respiratórias e patógenos causadores da doença infectocontagiosa. A quarta rota provável é a transmissão fecal-oral ${ }^{4}$.

A proteção respiratória é crucial para prevenir a transmissão dos patógenos virais. Dois tipos de máscaras são mais utilizados: as denominadas máscaras cirúrgicas e os respiradores do tipo peças faciais filtrantes (PFF). Para o senso comum, todos os aparatos utilizados com a finalidade de filtrar o ar inalado pela pessoa exposta são chamados de máscaras.

As máscaras cirúrgicas são utilizadas para proteger o paciente de secreções projetadas do nariz e da boca do profissional de saúde. Essas máscaras também oferecem barreira contra o contato do profissional com respingos de fluidos corporais do paciente (sangue, escarro, saliva, líquido cefalorraquidiano, entre outros), mas não oferecem vedação adequada da região da face e não foram projetadas ou certificadas para protegê-lo da exposição a aerossóis ${ }^{6}$.

Os respiradores PFF são distintos das máscaras cirúrgicas porque retêm aerossóis. A capacidade de retenção desses equipamentos de proteção diz respeito à eficiência de filtração de partículas com diâmetro médio de 0,3 $\mu \mathrm{m}$ testada em laboratório: 80\% (PFF1), 94\% (PFF2) e 99\% (PFF3) .

O tamanho das partículas potencialmente retidas pelos três filtros é o mesmo. A diferença entre eles é a capacidade de retenção, sendo o PFF3 o mais eficiente nesse quesito. Em síntese, o nível de proteção dos respiradores depende da eficiência de filtração.

Além da qualidade do material em termos de filtragem, o design é fundamental para o ajuste do respirador às características antropomórficas. A eficácia dos respiradores com filtro contra partículas aerotransportadas está condicionada ao ajuste perfeito da peça ao rosto do indivíduo, de maneira a proporcionar uma vedação facial estanque ${ }^{26,27}$. Se não for assim, o ar ambiente que carreia o material entrará em contato com o organismo do sujeito exposto. Nessa situação, denominada de vazamento, o ar não filtrado será conduzido até a região corporal que devia estar protegida pela peça. Tecnicamente, o ajuste correto do respirador é mais importante para a proteção contra materiais aerotransportados do que a capacidade de filtração do material. Porém, gestos necessários à execução das tarefas movimentam os equipamentos faciais. Além disso, desconforto e fadiga se configuram como limites para a vigília autoprotetiva. Dito de outro modo, o uso prolongado dos EPI pode levar a descontrole dos próprios gestos e aumento do desconforto térmico e tátil. Essas situações comprometem a proteção em tese garantida ${ }^{28}$. 
O nível de proteção do equipamento PFF2 (94\%) corresponde ao mínimo recomendado contra agentes biológicos em serviços de saúde. O protetor facial nomeado de N95, que atende aos padrões dos EUA, é considerado similar ao PFF2 porque retém pelo menos $95 \%$ das partículas inaláveis ${ }^{6}$.

Embora os modelos de PFF com válvula de exalação sejam mais confortáveis, não são recomendados. A válvula diminui a segurança porque o ar exalado do profissional de saúde é lançado para o ambiente e, se ele estiver contaminado, é passível de carrear os patógenos virais para o entorno ${ }^{6}$.

Em todos os protocolos analisados, consta um maior grau de proteção em casos de exposição a procedimentos geradores de aerossóis, como ressuscitação cardiopulmonar, em que pesem as diferenças quanto ao tipo de respirador recomendado. O Quadro 2 sintetiza a comparação entre os protocolos dos países selecionados. À exceção da Argentina ${ }^{16}$, que indica N95, os demais países incluem respiradores mais potentes em ambientes com aerossóis. Entre os protocolos nacionais, o da China ${ }^{14}$ é o único a recomendar modelos para o rosto inteiro nos casos de procedimentos de risco aumentado. Tal segurança potencializada, se tratando desse país, não é surpreendente, já que além de ter sido o cenário do primeiro surto da COVID-19, a China foi duramente atingida por epidemias anteriores ao novo coronavírus ${ }^{29}$.
A elevada carga viral no caso da COVID-19 desaconselharia o uso de máscaras cirúrgicas, em prol de outros tipos mais potentes, até mesmo em serviços onde não são realizados procedimentos invasivos. Mas os resultados das pesquisas ainda são limitados, explicando a falta de convergência entre os protocolos da amostra quanto ao tipo de equipamento de proteção respiratória ${ }^{4}$.

A reutilização de máscaras e respiradores não é um procedimento incluído nas diretrizes da $\mathrm{OMS}^{8}$. O protocolo brasileiro prevê, contudo, essa situação quando esclarece a necessidade de se respeitar as rotinas da comissão de infecção hospitalar do serviço de saúde, bem como seguir o protocolo de reutilização ${ }^{7}$ (Quadro 3).

A transmissão transocular do novo coronavírus é provável, justificando o uso de equipamento de proteção dos olhos. A especificação desse quesito é variável (óculos ou equipamentos tipo viseiras) nos protocolos estudados. Geralmente, os EPI oculares são usados nos serviços de assistência a pacientes com quadros clínicos graves.

Com relação às vestimentas de proteção, os modelos de capote com ou sem mangas compridas, por exemplo, e os materiais desses trajes - impermeáveis ou não - estão atrelados ao grau de proteção necessário. Alerta-se sobre os limites dos aventais aos objetivos de proteção, porque regiões do pescoço, das pernas e o dorso permanecem expostas ${ }^{30}$.

Quadro 2 Tipos de equipamentos de proteção individual incluídos nos protocolos de proteção dos profissionais dos serviços de saúde expostos ao novo coronavírus, de acordo com o país e com a Organização Mundial da Saúde, 2020

\begin{tabular}{|l|l|l|l|l|l|l|}
\hline \multicolumn{2}{|c|}{ Proteção } & \multicolumn{1}{|c|}{ OMS $^{8}$} & Argentina $^{16}$ & \multicolumn{1}{|c|}{ China $^{14}$} & Estados Unidos $^{15}$ & Brasil $^{7}$ \\
\hline \multirow{2}{*}{} & $\begin{array}{l}\text { Contato com } \\
\text { pacientes }\end{array}$ & $\begin{array}{l}\text { Máscara } \\
\text { cirúrgica }\end{array}$ & $\begin{array}{l}\text { Máscara } \\
\text { cirúrgica }\end{array}$ & PFF2 & $\begin{array}{l}\text { Máscara } \\
\text { cirúrgica }\end{array}$ & Máscara cirúrgica \\
\cline { 2 - 7 } & $\begin{array}{l}\text { Em caso de } \\
\text { aerossóis }\end{array}$ & $\begin{array}{l}\text { N95 ou } \\
\text { superior }\end{array}$ & N95 & PRRI/PRPA & N95 ou superior & N95 ou superior \\
\cline { 2 - 7 } & Reuso & $\begin{array}{l}\text { Não } \\
\text { menciona }\end{array}$ & Autorizado & Não menciona & Autorizado & Autorizado \\
\hline \multirow{2}{*}{ Corpo } & $\begin{array}{l}\text { Capote } \\
\text { Avental* }\end{array}$ & Capote & $\begin{array}{l}\text { Uniforme } \\
\text { Gorro }\end{array}$ & $\begin{array}{l}\text { Capote } \\
\text { Avental* }\end{array}$ & $\begin{array}{l}\text { Capote } \\
\text { Gorro }\end{array}$ \\
\hline Olhos & Propé & Óculos & $\begin{array}{l}\text { Óculos } \\
\text { PF }\end{array}$ \\
\hline Mãos & Óculos & $\begin{array}{l}\text { Óculos } \\
\text { PF }\end{array}$ & PF & Óculos & Luvas & Luvas \\
\hline
\end{tabular}

*Em caso de aerossóis; PFF2: Peça semifacial filtrante com eficiência mínima de filtração de 94\%; N95: Peça semifacial filtrante com eficiência mínima de filtração de 95\% (equivalente ao PFF2); PFF3: Peça semifacial filtrante com eficiência mínima de filtração de 99\%; PRRI: Proteção Respiratória de Rosto Inteiro; PRPA: Proteção Respiratória Purificadora de Ar; PF: Protetor Facial. 
Quadro 3 Tipos de equipamentos de proteção individual incluídos nos protocolos de proteção dos profissionais dos serviços de saúde expostos ao novo coronavírus: Brasil e estados do Amazonas, Bahia, Minas Gerais e São Paulo, 2020

\begin{tabular}{|c|c|c|c|c|c|c|}
\hline \multicolumn{2}{|c|}{ Proteção } & Brasil $^{7}$ & Amazonas $^{20}$ & Bahia $^{17}$ & Minas Gerais ${ }^{18}$ & São Paulo ${ }^{19}$ \\
\hline \multirow{3}{*}{ 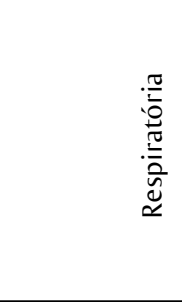 } & $\begin{array}{l}\text { Contato com } \\
\text { pacientes }\end{array}$ & $\begin{array}{l}\text { Máscara } \\
\text { cirúrgica }\end{array}$ & Máscara cirúrgica & Máscara cirúrgica & $\begin{array}{l}\text { Máscara } \\
\text { cirúrgica }\end{array}$ & Máscara cirúrgica \\
\hline & $\begin{array}{l}\text { Em caso de } \\
\text { aerossóis }\end{array}$ & $\begin{array}{l}\text { N95 ou } \\
\text { superior }\end{array}$ & N95 & N95 & N95 ou superior & N95 ou superior \\
\hline & Reuso & Autorizado & Sem menção & $\begin{array}{l}\text { Recomenda seguir } \\
\text { orientação do } \\
\text { fabricante }\end{array}$ & Sem menção & $\begin{array}{l}\text { Recomenda } \\
\text { seguir orientação } \\
\text { do fabricante }\end{array}$ \\
\hline Corpo & & $\begin{array}{l}\text { Capote } \\
\text { Gorro } \\
\end{array}$ & $\begin{array}{l}\text { Capote } \\
\text { Avental } \\
\text { Gorro }\end{array}$ & $\begin{array}{l}\text { Capote } \\
\text { Avental }\end{array}$ & $\begin{array}{l}\text { Capote } \\
\text { Avental } \\
\text { Gorro }\end{array}$ & $\begin{array}{l}\text { Capote } \\
\text { Avental } \\
\text { Gorro } \\
\end{array}$ \\
\hline Olhos & & $\begin{array}{l}\text { Óculos } \\
\text { PF }\end{array}$ & $\begin{array}{l}\text { PO } \\
\text { PF }\end{array}$ & $\begin{array}{l}\text { Óculos } \\
\text { PF }\end{array}$ & PF & $\begin{array}{l}\text { Óculos } \\
\text { PF }\end{array}$ \\
\hline Mãos & & Luvas & Luvas & Luvas & Luvas & Luvas \\
\hline
\end{tabular}

PFF2: Peça semifacial filtrante com eficiência mínima de filtração de 94\%, equivalente ao protetor N95, cuja eficiência é de 95\%; PFF3: Peça semifacial filtrante com eficiência mínima de filtração de 99\%; PF: Protetor Facial; PO: Protetor Ocular.

\section{A adesão dos profissionais aos EPI: entre imperativos contraditórios}

A escassez da oferta de EPI sob a excepcionalidade da COVID-19 foi registrada nos primeiros meses da pandemia. Cinquenta por cento dos médicos entrevistados no território brasileiro ( $\mathrm{n}=2.321$ ) estavam enfrentando falta de EPI no início do surto ${ }^{31}$. A escassez, entretanto, não foi o único problema nesse âmbito, pois a baixa adesão ao uso de EPI é uma questão recorrente ${ }^{32}$ que tem sido interpretada como "desconhecimento e desinteresse por parte dos sujeitos"33. Em contraponto, outras hipóteses sobre a baixa adesão aos EPI também são mencionadas na literatura especializada: pode ser um efeito das contradições entre as recomendações protetivas e a dimensão ética do cuidado aos pacientes ${ }^{34}$, ou uma resposta pragmática em situação de reconhecida ineficácia desses aparatos.

O relato dos profissionais traz elementos para a compreensão das racionalidades que embasam a adesão aos EPI durante o tratamento dos pacientes com COVID-19. Veja-se o caso de

um paciente de 64 anos obeso, que evoluiu com piora rápida do padrão respiratório [...]. Naquele momento, parte da equipe transportava outro paciente à UTI. Mesmo com a equipe reduzida, tivemos que realizar uma intubação. [...] Como os dois médicos usam óculos, durante o procedimento os óculos embaçaram com a combinação de máscara e face shield. Assim, não tivemos sucesso. Na segunda tentativa, um dos médicos retirou deliberadamente a proteção facial para enxergar melhor, e conseguiu realizar o procedimento ${ }^{35}$.
O comportamento do médico em retirar a proteção visual, longe de desconhecimento ou desinteresse, objetivou se livrar do embaçamento das lentes de seus óculos para visualizar as zonas nas estruturas da orofaringe e adjacências que orientam o posicionamento correto da lâmina do laringoscópio durante o procedimento da intubação orotraqueal.

Os exemplos convidam a refletir sobre as dimensões da atividade humana no trabalho. Em vez de orientado exclusivamente pela racionalidade instrumental ou administrativa no trabalho, o sujeito desenvolve sua atividade movido também por finalidades intrínsecas, que são elaboradas em sua relação com os outros em contextos específicos ${ }^{36}$. Compromissos éticos foram postos em confronto com as diretrizes de uso do EPI, pois os profissionais de saúde expressaram preocupações quanto às impressões dos pacientes diante da aparência do cuidador. Considerando que a estética da paramentação é inusual, um tipo de estranhamento pode levar o paciente a perceber, em vez de cuidado, uma espécie de aspereza no trato, além de concluir apressadamente sobre a gravidade e irreversibilidade do seu quadro clínico. Fissuras desse tipo na relação entre o profissional e o paciente são consideradas pelo pessoal da saúde, haja vista o reconhecimento sobre a influência negativa que acarretam sobre o sucesso terapêutico ${ }^{24}$.

\section{Abordagem sistêmica no lugar da resposta única}

A escassez de EPI para os profissionais de saúde da linha de frente tem sido destacada como um dos 
principais problemas no enfrentamento da COVID-19. Convém, no entanto, revisar os problemas acarretados pelo uso desses equipamentos, que embora fundamentais, não são suficientes para a proteção a que se destinam.

O uso do EPI não é inofensivo, pois interfere nas funções sensoriais do portador, tendo sido evidenciados déficits de comunicação, diminuição da acuidade visual e auditiva, fadiga e efeitos sobre o desempenho. Frequentemente são percebidos como desconfortáveis e até mesmo insuportáveis ${ }^{37}$.

A maioria das roupas projetadas para fornecer resistência a fluidos infecciosos durante a assistência a pacientes infectados promove aumento da temperatura corporal porque impede a troca de calor entre a superfície corpórea e o ambiente, o que é fundamental para o conforto térmico. Nessas situações, é comum o aumento da produção do suor, que pode se tornar veículo de condução das partículas virais de uma região do corpo que estava protegida - cabeça, por exemplo - para mucosas dos olhos ou da boca ${ }^{38}$.

Inúmeras perturbações do organismo são decorrentes do uso prolongado de EPI, como desconforto térmico, sudorese local e odor estranho, sensação de pressão facial, irritação e prurido na região de contato. Ensaios clínicos evidenciaram que nenhuma das máscaras faciais era perfeitamente ajustável em mais de um quarto dos participantes em função de suas características antropomórficas. Desajustes desse tipo provocam pressões localizadas nas regiões do crânio e face, que são relacionadas ao desencadeamento de cefaleias ${ }^{39}$. Como agravante, raramente é facultado ao trabalhador escolher a máscara que ele julgar mais adequada às suas características e necessidades ${ }^{40}$.

O risco de contaminação no momento do uso ou retirada/descarte dos equipamentos tem sido evidenciado. Estudos experimentais confirmam que os atos de desparamentação exigem alto nível de precisão para não gerar zonas de contaminação. A proficiência no encadeamento dos gestos precisos, entretanto, depende de treinamento intensivo ${ }^{41}$. Uma alternativa foi contar com uma área exclusiva para o procedimento. Sob supervisão, o padrão de operações, aos poucos, é ritualizado, de maneira a promover segurança e diminuir a ansiedade do profissional de saúde no final de sua jornada ou depois de finalizado um procedimento de alto risco ${ }^{42}$.

Resultados de estudos realizados nos cinco continentes identificaram que o apoio da gestão e a adequação da estrutura dos estabelecimentos influenciam na adesão às precauções ${ }^{24}$. A abordagem sistêmica embasa as recomendações do $\mathrm{MS}^{7}$ visando proteger, da COVID-19, os profissionais da saúde. Além de contemplar instruções para o uso adequado dos EPI, inclui o autocuidado em saúde mental, com ênfase nas condutas positivas de enfrentamento da sobrecarga emocional, derivada tanto da exposição a situações de sofrimento e morte quanto às pressões por resultado em um ambiente por vezes desprovido dos recursos materiais essenciais. Nesse tópico, estão dezenove itens relativos a medidas de apoio e de fortalecimento desses profissionais a serem conduzidas pelos líderes de equipe e gerentes, como buscar ajuda dos amigos e familiares ou reproduzir experiências bem-sucedidas que foram construídas em outras situações.

No âmbito da segurança ocupacional, o MS esclarece os objetivos de identificar e intervir nos fatores e situações de risco nos ambientes laborais. As medidas de engenharia para promover a proteção incluem desde instalações físicas e leiaute até provimento de dispensadores de soluções alcoólicas no ambiente de trabalho. Entre treze recomendações nesse tópico, citam-se a instalação de espaços de acolhimento e triagem, de maneira a facilitar o isolamento de pacientes suspeitos de COVID-19, unidade de isolamento respiratório com pressão negativa e filtro específico para procedimentos que podem gerar aerossóis ${ }^{7}$.

\section{Avanços e impasses na proteção dos profissionais dos serviços de saúde}

Determinadas pelas idiossincrasias individuais ou por fatores estruturais, a adesão ao uso de EPI faz parte de um contexto de saúde e segurança ocupacional ${ }^{43}$. Apesar da reconhecida gênese multidimensional do adoecimento no trabalho, as intervenções nesse campo têm reproduzido a concepção da "causa única" nas análises que evidenciam baixa adesão ao uso dos equipamentos. Encontra-se suficientemente fundamentado o conhecimento sobre os limites dos EPI quando os programas de implantação desses recursos não são acompanhados de outras medidas. Além disso, a prescrição de seu uso sem o zelo pelas medidas de prevenção de caráter estrutural (de engenharia, administrativas e organizacionais), incluídas no protocolo brasileiro ${ }^{7}$, é uma resposta isolada para um problema bastante complexo, como se trata a proteção da exposição a patógenos virulentos nos ambientes dos serviços de saúde.

O intenso confronto de valores entre a autoproteção e o cuidado aos pacientes continua invisível na maioria dos processos de avaliação da implantação dos EPI. Ora, trata-se de uma dimensão fundadora da atividade nos processos de trabalho em saúde. A necessidade de transformação dessa ótica para alcançar êxito nas ações de prevenção depende de mais investimentos, com vistas a desembaraçar nós críticos relacionados ao conhecimento sobre a atividade laboral que é 
fundamental na elaboração de medidas de transformação ${ }^{36,44}$. As orientações para uso de EPI são mais efetivas quando articuladas às vivências dos trabalhadores. Experiências anteriores indicaram a relevância do envolvimento reflexivo e participativo dos trabalhadores no planejamento das intervenções ${ }^{45}$.

Em suma, à luz da experiência no manejo das epidemias respiratórias que antecederam a COVID-19, conforme apresentado, convêm considerar a influência de fatores distais sobre os comportamentos de autoproteção dos profissionais, em vez de concentrar as recomendações nos fatores mais proximais ao indivíduo ${ }^{36,37}$.

\section{Conclusão}

A produção científica nos primeiros meses da crise pandêmica é expressiva. Os resultados obtidos são pistas para a formulação de ações de proteção dos profissionais dos serviços de saúde contra a exposição ao risco de infecção. Existem, contudo, pontos de dissenso entre os protocolos, conclamando debates interinstitucionais, interpaíses e interestaduais sobre modelos de prevenção.

Avanços em várias direções foram observados, mas as medidas de prevenção ainda carecem de reformulações, haja vista as tensões relacionadas à racionalidade econômica e administrativa, como é o caso da indicação para reuso de equipamentos.

Os primeiros meses de 2020 propiciaram muitos aprendizados em diferentes espectros da vida em sociedade. Pela frente, são muitos os desafios. Para construir um sistema de saúde resiliente face ao impacto da pandemia COVID-19 em escala planetária, será necessário fortalecer o ambiente social, de maneira a estimular agendas de pesquisa para a revisão dos protocolos de proteção dos profissionais da saúde.

\section{Contribuições de autoria}

Assunção AA, Simões MRL, Maia EG, Alcantara MA e Jardim R contribuíram de forma substancial na concepção do estudo, no levantamento, análise e interpretação dos dados, na elaboração, revisões críticas e aprovação da versão final deste manuscrito e assumem integral responsabilidade pelo trabalho realizado e o conteúdo publicado.

\section{Referências}

1. Adams JG, Walls RM. Supporting the health care workforce during the COVID-19 global epidemic. JAMA. 2020;323(15):1439-40.

2. Assunção AA, Maia EG, Jardim R, Araújo TM. . Incidence of reported flu-like syndrome cases in Brazilian health care workers in 2020 (March to June). Int J Environ Res Public Health. 2021;18(11):5952.

3. Huang L, Lin G, Tang L, Yu L, Zhou Z. Special attention to nurses' protection during the COVID-19 epidemic. Crit Care. 2020;24(1):120.

4. Thomas JP, Srinivasan A, Wickramarachchi CS, Dhesi PK, Hung YM, Kamath AV. Evaluating the national PPE guidance for NHS healthcare workers during the COVID-19 pandemic. Clinical Med. 2020;20(3):242-7.

5. Ali Y, Alradhawi M, Shubber N, Abbas AR. Personal protective equipment in the response to the SARSCoV-2 outbreak - a letter to the editor on "World Health Organization declares global emergency: a review of the 2019 novel coronavirus (COVID-19)" (Int J Surg 2020; 76:71-6). Int J Surg. 2020;78:66-7.

6. Nicolai SHA, Aquino JD, Ventura FF, Benevides EAS. Prevenção à COVID-19: proteção respiratória. São Paulo: Fundacentro; 2020.
7. Ministério da Saúde (BR). Recomendações de proteção aos trabalhadores dos serviços de saúde no atendimento de COVID-19 e outras síndromes gripais. Brasília, DF: Secretaria de Vigilância em Saúde; 2020.

8. World Health Organization (WHO). Infection prevention and control during health care when novel coronavirus (nCoV) infection is suspected: interim guidance [Internet]. Geneva: WHO; 19 mar 2020 [citado em 22 abr 2021]. Disponível em: https://www.who.int/publications/i/item/10665331495

9. Hammond JS, Eckes JM, Gomez GA, Cunningham DN. HIV, trauma, and infection control: universal precautions are universally ignored. J Trauma. 1990;30(5):558-61.

10. Mitchell R, Ogunremi T, Astrakianakis G, Bryce E, Gervais R, Gravel D, et al. Impact of the 2009 influenza A (H1N1) pandemic on Canadian health care workers: a survey on vaccination, illness, absenteeism, and personal protective equipment. Am J Infect Control. 2012;40(7):611-6.

11. Hu X, Zhang Z, Li N, Liu D, Zhang L, He W, et al. Self-reported use of personal protective equipment among Chinese critical care clinicians 
during 2009 H1N1 influenza pandemic. PLoS One. 2012;7(9):e44723.

12. Barati M, Bashirian S, Jenabi E, Khazaei S, KarimiShahanjarini A, Zareian S, et al. Factors associated with preventive behaviours of COVID-19 among hospital staff in Iran in 2020: an application of the protection motivation theory. J Hosp Infect. 2020;105(3):430-3.

13. Cochrane Special Collections. Coronavirus (COVID-19): infection control and prevention measures [Internet]. Hoboken: Wiley; 20 jul 2021 [citado em 22 abr 2021]. Disponível em: https:/www.cochranelibrary.com/collections/doi/ SC000040/full?contentLanguage $=\mathrm{en}$

14. Tingbo L, Yu L. Handbook of COVID-19 prevention and treatment [Internet]. Hangzhou: Zhejiang University School of Medicine; 2020 [citado em 5 jul. 2021]. Disponível em: https:/gmcc.alibabadoctor. $\mathrm{com} /$ prevention-manual/reader?pdf=Handbook\%20 of\%20COVID-19\%20Prevention\%20and\%20 Treatment\%20(Standard).pdf

15. Centers for Disease Control and Prevention (CDC). Interim Infection Prevention and Control Recommendations for Healthcare Personnel During the Coronavirus Disease 2019 (COVID-19) Pandemic [Internet]. Atlanta: CDC; 2020 [citado em 22 abr 2021]. Disponível em: https://www.cdc. gov/coronavirus/2019-ncov/hcp/infection-controlrecommendations.html\#print

16. Ministerio de Salud (AR). Recomendaciones para el uso de los EPP [Internet]. Buenos Aires; 6 fev 2020 [citado em 5 jul 2021]. Disponível em: https://www.argentina.gob.ar/salud/coronavirus/ recomendaciones-uso-epp

17. Bahia (Estado). Diretoria de Vigilância Sanitária e Ambiental. Medidas de prevenção e controle que devem ser adotadas durante a assistência aos casos suspeitos ou confirmados de infecção pelo novo coronavírus COVID -2019. Nota técnica n. 01/2020. Salvador: NECIH/COVIM/DIVISA; 2020.

18. Minas Gerais (Estado). Secretaria Estadual de Saúde. COVID-19: atualização técnica ao protocolo de infecção humana pelo SARS-COV-2 n. 03/2020. precaução e uso de equipamentos de proteção individual (EPI). Belo Horizonte: SES; 2020.

19. São Paulo (Estado). Secretaria Estadual da Saúde. Novo coronavírus (2019ncov): medidas de prevenção e controle de infecção a serem adotadas na assistência à saúde. São Paulo: SES; 2020.

20. Amazonas (Estado). Recomendações aos profissionais de saúde sobre EPI usados na prevenção ao COVID-19. Nota Técnica n. 08/2020. Manaus: CECISS/DIPRE/FVS-AM; 2020.

21. Gould DJ, Moralejo D, Drey N, Chudleigh JH, Taljaard M. Intervenções para aumentar a adesão à higiene das mãos durante os cuidados de saúde ao paciente. Cochrane Database Syst Rev. 2017;(9):CD005186.

22. Moralejo D, El Dib R, Prata RA, Barretti P, Correa I. Como melhorar a aderência dos profissionais de saúde às Precauções Padrão para o controle de infecções associadas aos cuidados de saúde. Cochrane Database Syst Rev. 2018;(2):CD010768.

23. Verbeek JH, Rajamaki B, Ijaz S, Sauni R, Toomey E, Blackwood B, et al. Equipamento de proteção individual para profissionais de saúde para prevenir doenças altamente contagiosas pela exposição a fluidos corporais contaminados. Cochrane Database Syst Rev. 2020;(5):CD011621.

24. Houghton C, Meskell P, Delaney H, Smalle M, Glenton C, Booth A, et al. Barreiras e facilitadores para a adesão dos profissionais de saúde às orientações de prevenção e controle de infecções (PCI) para doenças infecciosas respiratórias: uma rápida síntese qualitativa das evidências. Cochrane Database Syst Rev. 2020;(4):CD013582.

25. Thanh BYL, Laopaiboon M, Koh D, Sakunkoo $\mathrm{P}$, Moe H. Intervenções comportamentais para incentivar o uso de equipamento de proteção respiratória entre trabalhadores. Cochrane Database Syst Rev. 2016;(12):CD010157.

26. Associação Brasileira de Normas Técnicas (ABNT). ABNT NBR 13698:2011. Equipamento de proteção respiratória - peça semifacial filtrante para partículas [Internet]. São Paulo: ABNT; 2011 [citado em 22 abr 2021]. Disponível em: https:/ www.abntcatalogo.com.br/norma.aspx?ID=86730

27. Wilkinson IJ, Pisaniello D, Ahmad J, Edwards S. Evaluation of a large-scale quantitative respiratorfit testing program for healthcare workers: survey results. Infect Control Hosp Epidemiol. 2010;31(9):918-25.

28. Regli A, Sommerfield A, von Ungern Sternberg BS. The role of fit testing N95/FFP2/FFP3 masks: a narrative review. Anaesthesia. 2021;76(1):91-100.

29. Ip VH, Sondekoppam RV, Özelsel TJ, Tsui BC. Coronavirus disease 2019 (COVID-19) pandemic: international variation of personal protective equipment (PPE) and infection prevention and control (IPC) guidelines. Anesth Analg. 2020;131(21)113-4.

30. Associação Brasileira de Medicina de Emergência (Abramede), Associação Médica Brasileira (AMB), Conselho Federal de Enfermagem (Cofen), Colégio Brasileiro de Enfermagem em Emergência (Cobeem). Recomendações para prevenção e controle de exposição no atendimento a pacientes portadores de COVID-19 para profissionais do atendimento pré-hospitalar e transporte de pacientes [Internet]. [local desconhecido]; 2020 [citado em 22 abr 2021]. Disponível em: https://bit.ly/3dDOzem

31. Bocchini B. Coronavírus: pesquisa mostra que 50\% dos médicos acusam falta de EPI. Agência Brasil [Internet]. Brasília; 28 abr 2020 [citado em 22 abr 2021]. Disponível em: https://bit.ly/2QHjwVV

32. Savoia E, Argentini G, Gori D, Neri E, PiltchLoeb R, Fantini MP. Factors associated with access and use of PPE during COVID-19: a crosssectional study of Italian physicians. Plos one. 2020;15(10):e0239024. 
33. Guimarães EAA, Araújo GD, Bezerra R, Silveira RC, Oliveira VC. Percepção de técnicos de enfermagem sobre o uso de equipamentos de proteção individual em um serviço de urgência. Cienc Enferm. 2011;17(3):113-23.

34. Floyd DL, Prentice Dunn S, Rogers RW. A meta analysis of research on protection motivation theory. J Appl Soc Psychol. 2000;30(2):407-29.

35. Brandão Neto RA, Velasco ITV. Médicos da emergência do Hospital das Clínicas vivem carrossel de emoções: mesmo com a proteção adequada, o contágio pelo coronavírus é quase inevitável. Folha de S. Paulo. 9 mar 2020:Sec. B2.

36. Garrigou A, Baldi I, Dubuc P. Apports de l'ergotoxicologie à l'évaluation de l'efficacité réelle des EPI devant protéger du risque phytosanitaire : de l'analyse de la contamination au processus collectif d'alerte. PISTES. 2008;10-1.

37. Yassi A, Moore D, FitzGerald JM, Bigelow P, Hon MCY, Bryce E, et al. Research gaps in protecting healthcare workers from SARS and other respiratory pathogens: an interdisciplinary, multistakeholder, evidence-based approach. J Occup Environ Med. 2005;47(1):41-50.

38. Sprecher AG, Caluwaerts A, Draper M, Feldmann H, Frey CP, Funk RH, et al. Personal protective equipment for filovirus epidemics: a call for better evidence. J Infec Dis. 2015;212(suppl. 2):S98-S100.

39. Ong JJ, Bharatendu C, Goh Y, Tang JZ, Sooi KW, Tan YL, et al. Headaches associated with personal protective equipment - a cross sectional study among frontline healthcare workers during COVID 19. Headache. 2020;60(5):864-77.

40. Ribeiro LCM, Souza ACS, Neves HCC, Munari DB, Medeiros M, Tipple AFV. Influência da exposição a material biológico na adesão ao uso de equipamentos de proteção individual. Cienc Cuid Saude. 2010;9(2):325-32.

41. Edmond MB, Diekema DJ, Perencevich EN. Ebola virus disease and the need for new personal protective equipment. JAMA. 2014;312(23):2495-6.

42. Kwon JH, Burnham CAD, Reske KA, Liang SY, Hink T, Wallace MA, et al. Assessment of healthcare worker protocol deviations and self-contamination during personal protective equipment donning and doffing. Infect Control Hosp Epidemiol. 2017;38(9):1077-83.

43. Khan S, Nabi G, Han G, Siddique R, Lian S, Shi H, et al. Novel coronavirus: how things are in Wuhan. Clin Microbiol Infect. 2020;26(4):399-400.

44. Vilela RADG, Almeida IMD, Mendes RWB. Da vigilância para prevenção de acidentes de trabalho: contribuição da ergonomia da atividade.

Ciencia Saude Colet. 2012;17(10):2817-30

45. Loro MM, Bittencourt VLL, Zeitoune RCG. Pesquisa convergente assistencial: equipe de enfermagem compartilhando saberes sobre riscos ocupacionais e propondo interveções. REME Rev Min Enfer. 2017;21:1-8. 